\title{
Upregulation of microRNA-335 and microRNA-584 contributes to the pathogenesis of severe preeclampsia through downregulation of endothelial nitric oxide synthase
}

\author{
FENG JIANG $^{1 *}$, JIPENG LI ${ }^{2 *}$, GUOJUN WU ${ }^{3}$, ZHUO MIAO ${ }^{1}$, LINSHAN LU ${ }^{1}$, \\ GUOPING REN $^{4}$ and XIAOHONG WANG ${ }^{1}$
}

\author{
${ }^{1}$ Department of Gynecology and Obstetrics, Tangdu Hospital, The Fourth Military Medical University, \\ Xi'an, Shaanxi 710038; Departments of ${ }^{2}$ Gastrointestinal Surgery and ${ }^{3}$ Urology, Xijing Hospital, \\ The Fourth Military Medical University, Xi'an, Shaanxi 710033; ${ }^{4}$ Department of Hematology, \\ Tangdu Hospital, The Fourth Military Medical University, Xi'an, Shaanxi 710038, P.R. China
}

Received October 15, 2014; Accepted May 28, 2015

DOI: $10.3892 / \mathrm{mmr} .2015 .4018$

\begin{abstract}
The aim of the present study was to identify the differentially expressed microRNAs (miRNAs) in placenta from patients with preeclampsia, and examine their roles in the pathogenesis of preeclampsia in vivo and ex vivo. The placental expression levels of miRNAs were examined in tissue samples harvested from 20 patients with preeclampsia and 20 healthy control individuals. A total of 18 miRNAs were differentially expressed (12 upregulated and six downregulated) among the preeclampsia cases, compared with the controls. By further functional/pathway analysis, two significantly upregulated miRNAs, miR-335 and miR-584, were identified. These target endothelial nitric oxide synthase (eNOS), which has been repeatedly reported to be involved in the development of preeclampsia. The present study then verified eNOS as a target gene of miR-335 and miR-584 using a luceriferase assay, and confirmed the expression patterns of the two miRNAs and eNOS in preeclampsic and normal placentas. Additionally, to examine the function of miR-584 and miR-335 in human placenta, the present study transiently transfected the HTR8/Svneo cell line with miR-584 and miR-335 mimics or their inhibitors, and the results of a subsequent Transwell insert invasion assay revealed that miR-584 and miR-335 inhibited the migratory ability of the trophoblast cells, and that the effect was 'rescued' by overexpressed eNOS. These data revealed a negative regulatory role of miR-584 and
\end{abstract}

Correspondence to: Dr Feng Jiang, Department of Gynecology and Obstetrics, Tangdu Hospital, The Fourth Military Medical University, 569 Xinsi Road, Baqiao, Xi'an, Shaanxi 710038, P.R. China

E-mail: jfeng_22@163.com

*Contributed equally

Key words: microRNA-335, microRNA-584, endothelial nitric oxide synthase, trophoblast cell, preeclampsia
miR-335 in the migration of HTR-8/SVneo cells by targeting eNOS, and identified miR-584 and miR-335 as potential novel therapeutic targets in preeclampsia.

\section{Introduction}

Preeclampsia, a medical condition characterized by hypertension and proteinuria during pregnancy, can result from a wide spectrum of pathophysiological processes involving impaired migratory capability, endothelial dysfunction and systemic metabolic disorders (1-3). Placenta, as the site where the mediators of those pathophysiological processes are generated, is essential in the development of preeclampsia (4), therefore, placenta-based investigations may substantially improve current understanding of the mechanism underlying the disease. Increasing evidence has indicated that differentially expressed genes in the placenta significantly contribute to the pathogenesis of preeclampsia (5-8), however, the regulation of these genes remains to be fully elucidated.

MicroRNAs (miRNAs) are a class of $\sim 22$ nucleotide-long non-protein-coding RNAs, which can regulate gene expression by binding to the 3 ' untranslated region (UTR) of target gene messenger RNA (mRNA), resulting in translational repression and/or mRNA degradation (9). It is generally considered that $\sim 1 / 3$ human genes are regulated by microRNA (10), and that microRNA is key in cellular activities, including cell proliferation, migration and apoptosis (11-13). Since these activities have been reported to be disturbed in preeclampsia, miRNAs may be involved in the pathogenesis of preeclampsia $(14,15)$. To elucidate how miRNAs are involved in the pathogenesis of preeclampsia, the present study investigated global placental expression levels of miRNA in preeclampsic and normal placentas using microarray analysis, and selected the two most significantly upregulated miRNAs, miR-355 and miR-584, for further functional analysis due to their reported involvement in the development of preeclampsia $(14,15)$ or the roles in the regulation of cell proliferation and migration $(16,17)$. By in-silicon analysis and miRNA database searching, the 
present study aimed to identify the shared target gene of miR-355 and miR-584.

Considering the fact that aberrant expression of endothelial nitric oxide synthase (eNOS) may compromise the migratory capability of trophoblast cells (18), and that a lack of eNOS may directly exacerbate the preeclampsia-like phenotype by interfering with the endothelin system (19), the present study hypothesized that miR-335 and miR-584 may function as a regulator of trophoblast cell behavior by targeting eNOS. To assess this hypothesis, the effect of overexpressed miR-335 and miR-584 on the proliferation and invasion of trophoblast cells, and the potential 'rescue' effect by eNOS were investigated.

\section{Materials and methods}

Study population and data collection. The placenta microarray investigation population in the present study comprised 20 healthy pregnant females (age, 28.35 \pm 6.34 years) and 20 patients with severe preeclampsia (age, 28.67 \pm 7.12 years), recruited from the Department of Gynecology and Obstetrics, Tangdu Hospital, The Fourth Military Medical University (Xi'an, China). Normal pregnancy was defined as a previously and currently normotensive pregnant female, who delivered a healthy neonate following 37 weeks of gestation. Severe preeclampsia was defined as individuals with sustained $(\geq 2$ measures, 6 h apart) blood pressure elevation $(>160 / 110 \mathrm{mmHg})$ following 20 weeks of gestation, combined with significant proteinuria, which was defined as the sustained ( $\geq 2$ measures, $4 \mathrm{~h}$ apart) presence of elevated protein in the urine $(>30 \mathrm{mg} / \mathrm{dl}$ or $>1+$ on a urine dipstick). The study was approved by the Research Ethic Committees at The Fourth Military Medical University, and written consent was obtained from all subjects. Individuals with a history of renal disease, spontaneous abortion, gestational diabetes, or fetal chromosomal or congenital abnormalities were excluded from the investigation. Placental sample collection was performed, as described previously (5). Briefly $\sim 0.5 \mathrm{~cm}^{3}$ placental tissue was obtained via biopsy from 16 sites, including eight maternal and eight fetal sides, to achieve uniformity and adequate sampling. The tissue samples obtained were plaved into RNAlater (Qiagen Inc., Valencia, CA, USA) and stored at $-80^{\circ} \mathrm{C}$.

Total RNA isolation. All tissue samples from the same placenta were pooled together, and total RNA was extracted using TRIzol reagent (Invitrogen Life Technologies, Carlsbad, CA, USA), according to the manufacturer's instructions, with little modification. The purity of the isolated RNA was determined using a spectrophotometer (6310; Jenway, Ltd, Essex, UK). RNA integrity was confirmed using electrophoresis on an agarose gel (Sigma-Aldrich, St. Louis, MO, USA).

miRNA microarray analysis. Total RNA (5 $\mu \mathrm{g})$ from each sample was used in the microarray expression profiling assay. An miRCURY ${ }^{\mathrm{TM}}$ Array Labeling kit (Exiqon, Vedbaek, Denmark) was used to label the miRNAs, and the labeled RNA samples were hybridized to the miRNA microarray chip (Exiqon, Vedbaek, Denmark). The microarray set consisted of 1,035 probes, including 455 probes against human mature microRNA sequences, 344 probes against mouse miRNAs and 236 probes against rat miRNAs. A GenePix 4000B laser scanner (Axon Instruments, Foster City, CA, USA) was used to obtain the hybridization data, and the images were digitized and analyzed using GenePix 4.0 software (Axon Instruments).

Cell lines and cell culture. The HTR8/Svneo cells (1x10 $)$ were cultured in RPMI-1640 (Invitrogen Life Technologies), supplemented with $10 \%$ fetal bovine serum, $100 \mathrm{U} / \mathrm{ml}$ penicillin and $100 \mathrm{mg} / \mathrm{ml}$ streptomycin (Invitrogen Life Technologies).

Reverse transcription-quantitative polymerase chain reaction $(R T-q P C R)$ analysis. The target gene of the miRNA was predicted using miRanda (www.miranda.org) and TargetScan (www.targetscan.org), and the relevant signaling pathway was analyzed using DIANA (http://diana.cslab.ece.ntua.gr/pathways/) and KEGG (http://www.genome.jp/kegg/pathway/hsa/ hsa05200.html). The total RNA was isolated from the cultured cells or placental tissues using TRIzol ${ }^{\circledR}$ reagent (Invitrogen Life Technologies), according to the manufacturer's instructions. PrimeScript RT Reagent kit (Takara Biotechnology, Co., Ltd., Dalian, China) was used to reverse transcribe RNA for RT-qPCR analysis. A total of $20 \mu \mathrm{g}$ cDNA was used as a template for amplification of miRNA or mRNA using SYBR Premix Ex Taq ${ }^{\mathrm{TM}}$ II (Takara Biotechnology Co., Ltd.) under the following conditions: $95^{\circ} \mathrm{C}$ for $30 \mathrm{sec}$, followed by 40 cycles at $95^{\circ} \mathrm{C}$ for $5 \mathrm{sec}$ and $60^{\circ} \mathrm{C}$ for $30 \mathrm{sec}$, then a final extension step at $72^{\circ} \mathrm{C}$ for $5 \mathrm{~min}$. RT-qPCR was performed using the following primer sets from Sangon Biotech Co., Ltd. (Shanghai, China): Forward 5'-ACACTCCAGCTGGGTCAAGAGCAATAA CGAAA-3' and reverse 5'-CTCAACTGGTGTCGTGGA-3' for miR-335, and forward 5'-CAGGGTTGCTGTTACTGG AG-3' and reverse 5'-AATCTAACCCCACATTTCCCC-3' for miR-584. The following primer set: Forward 5'-CTCGCT TCGGCAGCACA-3' and reverse 5'-AACGCTTCACGAATT TGCG T-3' was used for U6 detection, and Forward 5'-CCC TTCAGTGGCTGGTACAT-3' and reverse 5'-CACGATGGT GACTTTGGCTA-3' was used for eNOS detection. A SYBR Green real time detection system (Bio-rad Laboratories, Inc., Hercules, CA, USA) was used to quantitatively measure the levels of expression. U6, an internal control, was used to normalize the expression levels of miR-335, miR-584 and eNOS.

Western blot analysis. The cells were lysed using lysis buffer supplemented with protease inhibitor (Sigma-Aldrich). The protein samples (20 ng) were loaded onto an $10 \%$ SDS-PAGE gel, and the separated proteins were then transferred onto a polyvinylidene difluoride membrane (EMD Millipore, Billerica, MA, USA) prior to being blocked with 10 mMTris- $\mathrm{Cl}$ (pH 8.0), $150 \mathrm{mMNaCl}$ and $0.05 \%$ Tween 20 (TBST) containing 5\% nonfat dry milk powder at room temperature for $1 \mathrm{~h}$. The membrane was subsequently incubated with rabbit anti-human eNOS polyclonal antibody (1:1,000; cat. no. ab5589; Abcam, Cambridge, MA, USA) at room temperature for $2 \mathrm{~h}$, followed by incubation with the goat anti-rabbit secondary antibody (1:10,000; ab6721; Abcam) at room temperature for another $2 \mathrm{~h}$. An enhanced chemiluminescence kit (Pierce Biotechnology, Inc., Rockford, IL, USA) was used to detect chemical fluorescence, according to the manufacturer's instructions. The relative density of the bands were analyzed and normalized by $\beta$-actin. 
Plasmids, small interfering (si)RNA and transfection. The siRNA, duplex against human eNOS (anti-eNOS-siRNA), miR-335 and miR-584 mimics, miR-335 and miR-584 inhibitors (anti-miR-335 and anti-miR-584) and scramble control were purchased from Ambion Life Technologies (Austin, TX, USA). The coding sequence of eNOS was PCR amplified (PCR conditions: $95^{\circ} \mathrm{C}$ for $30 \mathrm{sec} ; 30$ cycles at $95^{\circ} \mathrm{C}$ for $1 \mathrm{~min}, 58^{\circ} \mathrm{C}$ for $30 \mathrm{sec}$ and $68^{\circ} \mathrm{C}$ for $2 \mathrm{~min}$; and $72^{\circ} \mathrm{C}$ for $5 \mathrm{~min}$ ) and inserted into the pcDNA4.0 vector (Invitrogen Life Technologies) to produce a construct expressing eNOS in trophoblast cells.

Luciferase assay. The HTR-8/SVneo cells were cultured to $70 \%$ confluence and transfected with different constructs (pRL-SV40; Promega Corporation, Fitchburg, WI, USA), containing the wild-type or mutant eNOS 3'UTR, pRL-TK, and miR-584 or miR-335 mimics. A QuickChange XL site-directed mutagenesis kit (Stratagene, La Jolla, CA, USA) was used to introduce the variant. The cells were collected after $24 \mathrm{~h}$ at room temperature and the activities of either firefly or Renilla luciferase were determined using an LB 955 Luminometer system using the dual luciferase reporter system (Promega Corporation), according to the manufacturer's instructions. The activity of firefly luciferase was normalized to that of Renilla luciferase.

Cell survival and proliferation assay. Cell survival was evaluated using a 3-(4,5-dimethylthiazol-2-yl)-2,5-diphenyltetrazolium bromide (MTT) assay. The cells were seeded in 96-well plates at a density of 800 cells/well and were incubated for 24, 48 and $72 \mathrm{~h}$. MTT $(5 \mathrm{mg} / \mathrm{ml} ; 20 \mu \mathrm{l}$; Sigma-Aldrich) was added to each well and incubated for $4 \mathrm{~h}$, and the supernatants were removed, followed by the addition of $150 \mathrm{ml}$ dimethyl sulfoxide (Sigma-Aldrich). The absorbance value (optical density; OD) of each well was measured at $490 \mathrm{~nm}$ using a Jenway 6310 spectrophotometer. The experiments were performed three times.

Transwell invasion and migration assays. A Transwell insert invasion assay was performed in 24-well fitted inserts with membranes ( $8 \mathrm{~mm}$ pore size; Costar; Corning Incorporated, New York City, NY, USA). The cells transfected with the control, miRNA mimics, miRNA inhibitors or eNOS were treated with $10 \mathrm{mg} / \mathrm{ml}$ mitomycin C (Sigma-Aldrich) for $2 \mathrm{~h}$ and added to the top of the wells at the density of $2.6 \times 10^{5}$ cells/ well. Cell invasion was examined using a polycarbonate membrane cell culture insert, which was coated with growth factor-reduced Matrigel (Corning incorporated, Corning, NY, USA). The invaded cells on the lower surface of the membrane were stained with trypan blue (Sigma-Aldrich) and counted under a microscope (CX22; Olympus Corporation, Tokyo, Japan), and the invasion index was calculated using the following equation: Invasion index $=$ treated $/ \operatorname{control} \%$. Experiments were performed three times.

Statistical analysis. Raw data were normalized using GenePix 4.0 software (Axon Instruments, Foster City, CA), and median centered using the Bioconductor package (www. bioconductor. org). SAM software (http://www.stat.stanford.edu/tibs.SAM) was used to determine the differentially expressed miRNAs in the severe preeclampsia samples, which comprised genes with a significant $(\mathrm{P}<0.05)$ differential expression of $\geq 1.5$-fold. The Transwell insert invasion assay, proliferation assay, RT-qPCR, western blotting and luciferase assay were repeated at least three times, and the results are expressed as the mean \pm standard deviation. Student's t-test or one-way analysis of variance was used to detect differences, and statistical analysis was performed using SPSS software (version 17.0; SPSS, Inc., Chicago, IL, USA). $\mathrm{P}<0.05$ was considered to indicate a statistically significant difference.

\section{Results}

miRNA microarray analysis. The placenta microarray performed in the present study was compared between 20 healthy pregnant females and 20 patients with severe preeclampsia. No significant difference was present between the normal pregnant and the preeclamptic females in the present study regarding maternal age, body mass index, glucose tolerance, infant birth weight or gestational age (Table. I). Based on the evaluation of fold change (absolute fold change $>1.5$ ) and statistical analysis $(\mathrm{P}<0.05), 18$ miRNAs were significantly differentially expressed among the preeclampsia cases, compared with the controls, which included 12 upregulated and six downregulated miRNAs (Fig. 1). Of these, the two most significantly upregulated miRNAs, miR-355 and miR-584, were selected for further functional analysis due to their indicated involvement in preeclampsia pathogenesis $(14,15)$ or reported regulatory roles in cell proliferation and migration $(16,17)$. The results from confirmatory RT-qPCR experiments revealed the mRNA expression levels in the preeclampsia cases and controls for the selected miRNAs (Fig. 2A and B), and the fold changes in expression levels identified in the RT-qPCR experiments were comparable with the preceding microarray experiment.

Identification of eNOS as a shared target gene of miR-355 and miR0-584. The present study then performed searches using miRanda and Targetscan for the potential target genes, to identify the mediators of miR-335 and miR-584. By screening the resulting candidate genes based on their functions, combined with information about the microRNA using the DIANA Lab-based microRNA pathway analysis tool (http://diana.cslab.ece.ntua. gr/pathways/) and the KEGG database (http://www.genome. $\mathrm{jp} / \mathrm{kegg} /$ pathway/hsa/hsa05200.html), the predicted genes were categorized functionally to narrow down the candidate genes, which were functionally associated with the regulation of trophoblast cell behavior or preeclampsia pathogenesis. The resulting gene identified was eNOS, a gene that has been repeatedly reported to be compromise the migratory capability of trophoblast cells (10), or directly exacerbate preeclampsia-like phenotype by interfering with endothelin system (12). The microRNAs, and their seed sequences in the 3'-UTR of eNOS, are presented in Fig. 1A and B. The present study demonstrated that overexpression of the miR-335 and miR-584 mimics, but not the control mimics, markedly repressed the activity of luciferase, when fused with the wild-type 3'-UTR of eNOS, but had minimal effect on the luciferase activity when fused with the mutated 3'-UTR of eNOS, as shown in Fig. 1C and D.

mRNA and protein expression levels of miR-335 and miR-584 are increased in preeclampsic placenta. As shown in Fig. 2A 
Table I. Clinical characteristics of pregnant healthy control and preeclamptic patients.

\begin{tabular}{lccc}
\hline Variable & Control $(\mathrm{n}=20)$ & Preeclampsia $(\mathrm{n}=20)$ & P-value \\
\hline Maternal age (years) & $26.3 \pm 5.2$ & $28.1 \pm 4.8$ & 0.262 \\
Blood pressure (mmHg) & & & \\
Systolic & $116 \pm 12.1$ & $169 \pm 11.2$ & $<0.001$ \\
Diastolic & $83 \pm 9.5$ & $111 \pm 10.1$ & $<0.001$ \\
24 h urine protein $(\mathrm{g})$ & $0.01 \pm 0.03$ & $4.36 \pm 2.32$ & $<0.001$ \\
Body mass index $\left(\mathrm{kg} / \mathrm{m}^{2}\right)$ & $25.8 \pm 4.1$ & $25.9 \pm 4.5$ & 0.942 \\
Infant birth weight $(\mathrm{g})$ & $3,578 \pm 243$ & $3,462 \pm 215$ & 0.118 \\
Placenta weight $(\mathrm{g})$ & $533 \pm 72$ & $513 \pm 61$ & 0.349
\end{tabular}

Data are expressed as the mean \pm standard deviation.

A

$\begin{array}{cc}\text { eNOS 3'UTR1 } & 5^{\prime} \text {-AGCCCCGCUCCUCCCCUCUUGAG-3' } \\ \text { miR-335 } & \text { IIIIIII } \\ \text { 3' -UGUAAAAAGCAAUAACGAGAACU-5' }\end{array}$

eNOS 3'UTR2

$\operatorname{miR}-584$
B

LUC-eNOS 3'UTR1

Luc-eNOS 3'UTR1 mutant

Luc eNOS 3'UTR2

Luc eNOS 3'UTR2 mutant

\begin{tabular}{|c|c|c|}
\hline \multicolumn{3}{|c|}{ GUCUUGA } \\
\hline Luciferase & 3'UTR & PolyA \\
\hline \multicolumn{3}{|c|}{ GAGAACU } \\
\hline Luciferase & उUTK & PolyA \\
\hline
\end{tabular}

\begin{tabular}{|c|c|c|}
\hline \multicolumn{3}{|c|}{ ACCAUAA } \\
\hline Luciferase & उंUंर & PolyA \\
\hline & IGGUAI & \\
\hline Luciferase & 3'UTR & PolyA \\
\hline
\end{tabular}

C

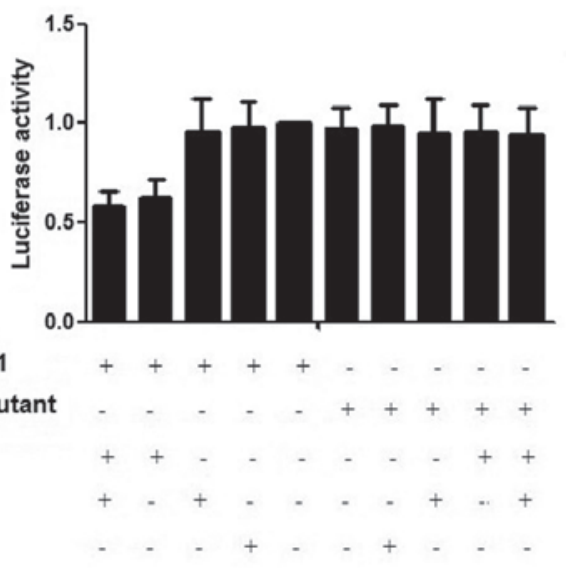

miR-negative
D

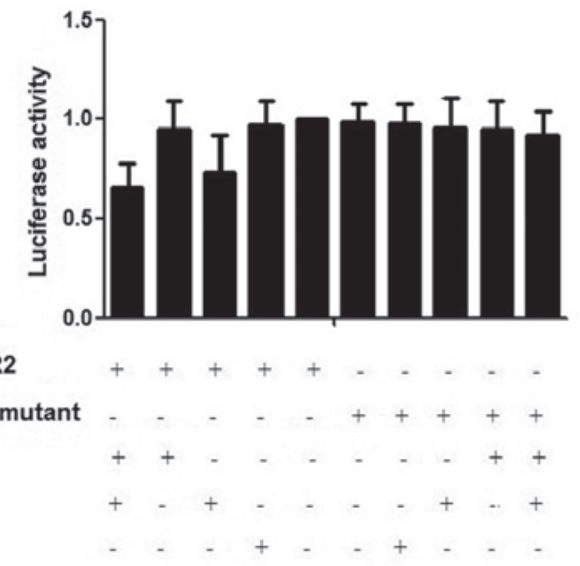

Luc eNOS 3'UTR2

Luc eNOS 3'UTR2 mutant

miR-335

miR-584

miR-negative

E

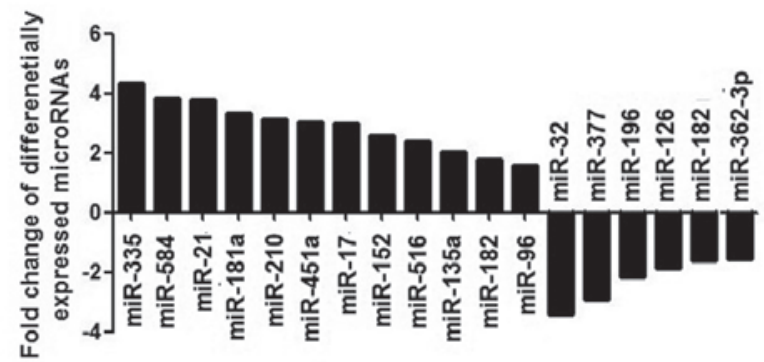

Figure 1. (A) Comparison of sequences between miR-335/miR-584 and the 3'UTR of eNOS (gray area represents seed sequence); (B) Comparison between the wild-type and mutant 3'UTR of eNOS; (C and D) Luciferase assay revealed that miR-335 and miR-584 suppressed the expression of the target gene by binding different seed sequences in the 3'UTR of eNOS; (E) Comparison in the fold change of differentially expressed miRs in the preeclampsia cases, compared with the controls. Data are expressed as the mean \pm standard deviation. miR, microRNA; UTR, untranslated region; eNOS, endothelial nitric oxide synthase; Luc, luciferase. 
A

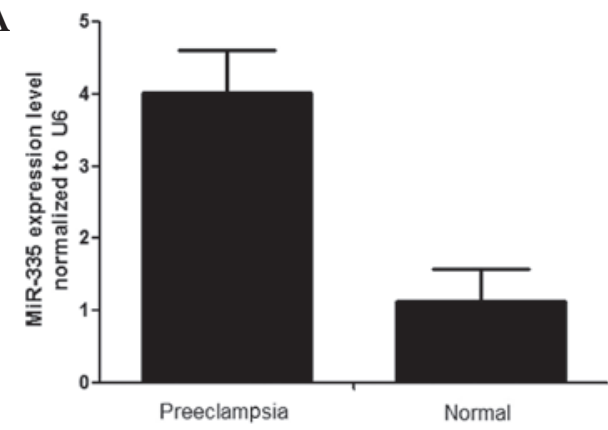

C

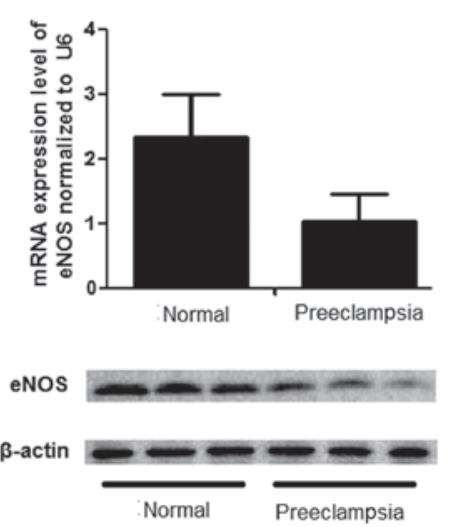

B

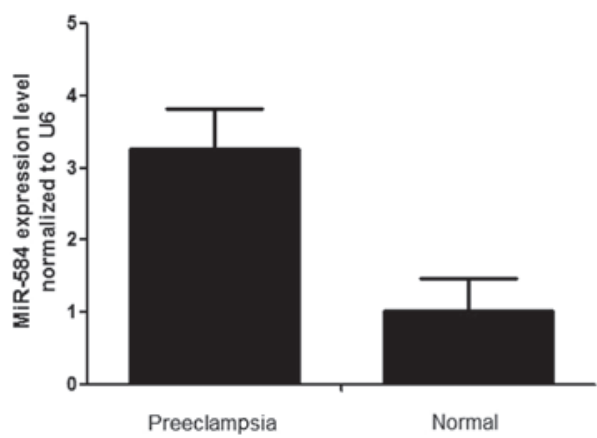

D

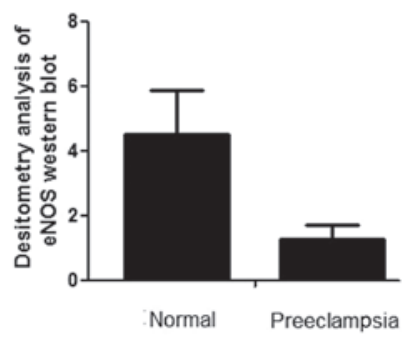

Figure 2. Comparison of expression patterns of miR-335/miR-584 and eNOS between 20 preeclampsic and 20 normal placentas. (A) mRNA expression of miR-335 in 20 preeclamptic placentas and 20 normal placentas; (B) mRNA expression of miR-584 in 20 preeclamptic placentas and 20 normal placentas; (C) mRNA expression of eNOS between 20 preeclamptic and 20 control placentas. (D) Upper panel, selected results of western blot analysis, (n=3/group); lower panel, densitometric analysis of western blotting results to determine protein levels of eNOS in placentas from 20 preeclamptic patients and 20 control individuals. Data are expressed as the mean \pm standard deviation. miR, microRNA; eNOS, endothelial nitric oxide synthase.

and $\mathrm{B}$, the relative expression levels of miR-335 and miR-584 in the preeclampsic placentas were significantly upregulated to $\sim 400 \%$ and $320 \%$, respectively, compared with normal placentas. In addition, the mRNA expression levels were measured uisng RT-qPCR, which revealed that the mRNA expression level of eNOS in the placentas derived from severe preeclampsic women was significantly lower than that observed in the normal placentas (Fig. 2C). The protein levels of eNOS were also examined using western blot analysis, and the relative density of eNOS in the tissue samples from severe preeclampsic placentas was $\sim 4.5$-fold higher than in the control, as shown in Fig. 2D and E.

miR-335 and miR-584 inhibit the migration of trophoblast cells. In addition, the HTR8/SVneo cells were transfected with either miR-335 and miR584 mimics or their inhibitors to further investigate the effects of miR-335 and miR-584 on trophpoblast cell behavior. Inhibitory effects of miR-335 and miR-584 on the expression levels of eNOS in the HTR8/SVneo cells were observed, and the inhibitory effect of specific anti-eNOS siRNA were similar to that of miR-335 or miR-584. The overexpression of miR-335 and miR584 markedly suppressed the migratory capability of the HTR8/SVneo cells individually and synergically (Fig 3D), however, the effect on the proliferation of the cells was minimal (data not shown).

eNOS rescues the inhibition of migration by miR-335 and miR-584 in trophoblast cells. To assess whether eNOS was directly involved in the inhibitory effect of miR-335 and
miR-584, the present study transfected eNOS (pcDNA4-eNOS) into the HTR8/SVneo cells overexpressing miR-335, miR-584 or anti-eNOS-siRNA, respectively. The results indicated overexpression of eNOS not only completely inhibited the effect of anti-eNOS-siRNA, miR-335/miR-584 in the HTR8/SVneo cells, but it also 'overcorrected' the migratory capability in those cells (Fig. 3).

Inhibition of miR-335 and miR-584 increases the migration of trophoblast cells. To further confirm the function of miR-335 and miR-584, a 'loss-of-function' assessment was performed by transfecting the HTR8/SVneo cells with miR-335 inhibitors and miR-584 inhibitors. The expression levels of miR-335 and miR-584 were significantly reduced $48 \mathrm{~h}$ after transfection with the inhibitors (Fig 3A and B). As expected, the migratory ability of the HTR8/SVneo cells transfected with the miR-335 inhibitors and miR-584 inhibitors were markedly higher than in the control, and was even higher in the cells transfected with both inhibitors (Fig 3C).

\section{Discussion}

miRNAs have emerged as potential novel diagnostic and therapeutic biomarkers for various medical disorders, including cancer $(20)$, pregnancy $(21,22)$ and tissue injury $(23)$. Preeclampsia is a life threatening medical condition complicated by hypertension and proteinuria during pregnancy, and is the most common cause of maternal mortality, morbidity, perinatal mortality and intrauterine growth retardation (24). 
A

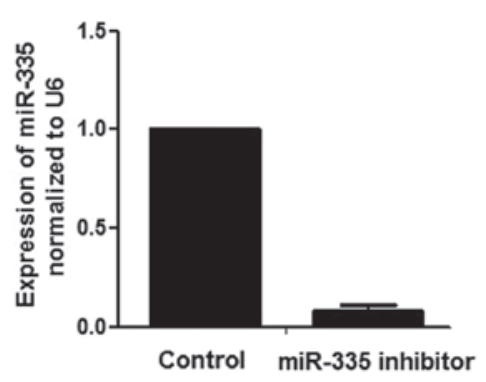

C

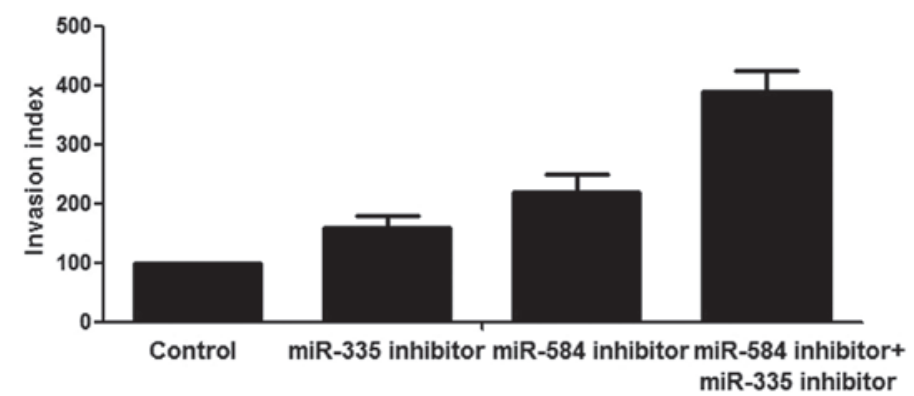

eNOS

$\beta$-actin miR-335 inhibitor
B

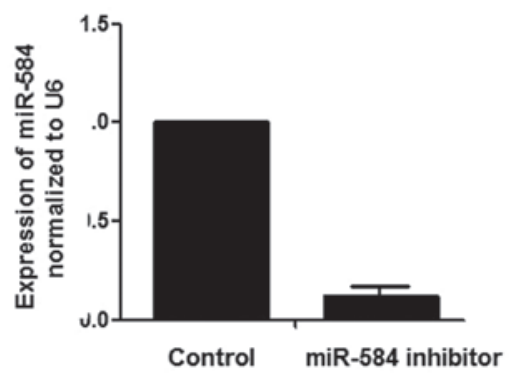

D

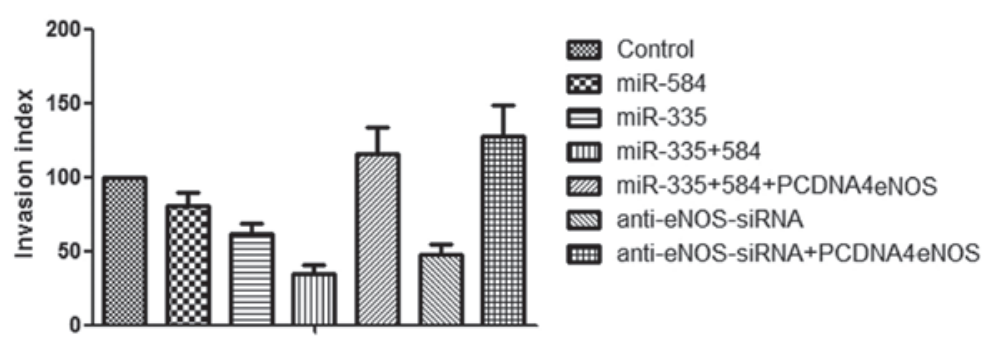

Figure 3. (A) Suppression of miR-335 by its inhibitor, anti-miR-335, in HTR8/SVneo cells; (B) Suppression of miR-584 by its inhibitor, anti-miR-584, in HTR8/SVneo cells. (C) Transwell insert assay to examine cell invasiveness following transfection with the two inhibitors and control. (D) Effect of the upregulation of miR-335/miR-584 on cell invasion in the HTR8/SVneo cells; upper panel, suppression of eNOS by miR-335/miR-584 mimics and anti-eNOS siRNA in the HTR8/SVneo cells. The histogram represents results of a Transwell insert assay to examine cell invasiveness following transfection of HTR8/SVneo cells with control, miR-335 mimic, miR-584 mimic, siRNA for eNOS and anti-eNOS siRNA. A 'rescue' effect was observed by the overexpression of eNOS in the HTR8/SVneo cells transfected with the miR-335/miR-584 mimics and anti-eNOS siRNA. Data are expressed as the mean \pm standard deviation. miR, microRNA; eNOS, endothelial nitric oxide synthase; siRNA, small interfering RNA.

Despite progression in understanding of this medical disorder, the mechanisms underlying preeclampsia remain to be fully elucidated (25). Investigation of the roles of differentially expressed miRNAs in the placenta of patients with preeclampsia are required to advancu current understanding of the pathogenesis of preeclampsia, and improve its diagnosis and management.

Abnormal development of the placenta is one of the major causes of preeclampsia (26), and delivery of the placenta remains the only definitive treatment for preeclampsia (27). Several studies (28-30) have examined the differential expression of miRNAs in preeclampsic placentas. In the present study, the differential expression of miRNAs in tissue samples derived from preeclampsic placentas were compared with those from normal controls. In total, 12 miRNAs were found to be significantly upregulated and six miRNAs were significantly downregulated in patients with severe preeclampsia. Of the identified miRNAs, miR-355 and miR-584 were selected for further functional analysis due to their indicated involvement in preeclampsia pathogenesis $(14,15)$ or reported regulatory roles in cell proliferation and migration $(16,17)$, and eNOS was identified as a shared target gene of miR-355 and miR-584.

Previously, the preeclampsia susceptibility locus has been mapped to chromosome 7q35-36 and eNOS has been identified as one of the susceptible genes of preeclampsia by fine mapping (31). A common variant in eNOS predisposes carriers to preeclampsia by promoting degradation of the enzyme (32). eNOS knockout leads to elevation of blood pressure and reduces the production of NO (33). Consistently, decreased maternal eNOS/NO exacerbates preeclampsia-like phenotype (19), and a significant reduction in the expression of eNOS is observed in the umbilical vessel of pregnant female with preeclampsia (34). Since eNOS was confirmed as a shared target of miR-335 and miR-584, the present study 
examined the effect of the two molecules on trophoblast cells. The results of this investigation revealed that miR-335 and miR-584 suppressed the expression of eNOS in the trophoblast cells, individually and synergically, and the expression pattern of miR-335, miR-584 and eNOS in the preeclampsic and normal placentas was examined. Consistent with the microarray results, significantly higher levels of miR-335 and miR-584, and a lower levels of eNOS were detected in the 20 preeclampsic placentas, compared with the 20 normal controls.

It is generally accepted that trophoblast cells contain various cytokines and biochemical effectors that are essential for metastatic invasion, a crucial process for the successful implantation and penetration of the endometrial stroma and blood vessels, which is necessary for a mature placenta and a viable fetus (35). Impaired trophoblast invasiveness may result in compromised placental perfusion during pregnancy, which is considered to be associated with various pathological processes, including fetal growth retardation, preeclampsia and spontaneous abortion (36). Accumulating evidence has indicated that inhibition of NO may cause preeclampsia-like conditions. Buhimschi et al demonstrated that administration of L-NAME, which competes with L-arginine and inhibits NO synthesis, to pregnant rats results in a preeclampsia-like condition. Additionally, administration of L-arginine to rats infused with L-NAME reversed the preeclampsia-like symptoms, which were observed in the pregnant rats treated with L-NAME alone (37).

Consistent with the data of the present study, miR-335 and miR-584 have repeatedly been observed to be differentially expressed in previous preeclampsia microarray studies $(14,15,38)$, and the two miRNAs have been demonstrated to be significantly associated with the invasive and migratory capabilities of human cells $(39,40)$. In the present study, miR-335 and miR-584 suppressed the migration and invasion of trophoblast cells via the regulation of eNOS. To investigate the function of miR-335 and miR-584 in vitro, miR-335 and miR-584 mimics or their inhibitors were transfected into HTR8/Svneo cells. miR-335 and miR-584 was observed to have an inhibitory effect on trophoblast cell migration and invasion individually, and the combined effect of co-transfection of the two small molecules was even more marked. Similarly, downregulation of miR-335 and miR-584 promoted trophoblast cell migration and invasion, individually and synergically, suggesting that endogenous miR-335 and miR-584 are involved in regulating cell behavior. In addition, the inhibitory effect of miR-335, miR-584 and anti-eNOS-siRNA on the migration of trophoblast cells was 'rescued' by overexpression of eNOS, however, simultaneous 'overcorrection' was observed in the microRNA and siRNA groups (Fig. 3D). As cGMP, the major downstream messanger of NO, also mediates migration in human cells (41), the present study hypothesized that the accumulation of cGMP, generated by intracellular overexpression of $\mathrm{NO}$, may be responsible for this overcorrection in the migratory ability of the cells.

Taken together, the data of the present study revealed for the first time, to the best of our knowledge, that miR-335 and miR-584 exert an inhibitory effect in regulating the migration and invasion of trophoblast cells through targeting eNOS, which may contribute to the pathogenesis of preeclampsia. These results advance current understanding of trophoblast disease, and miR335 and miR-584 may offer potential as novel therapeutic targets in the treatment of preeclampsia.

\section{Acknowledgements}

The present study was fully sponsored by the Natural Science Foundation of Shanxi Province (China; grant. no. 2014JM4133).

\section{References}

1. Sibai B, Dekker G and Kupferminc M: Pre-eclampsia. Lancet 365: 785-799, 2005.

2. Redman CW and Sargent IL: Latest advances in understanding preeclampsia. Science 308: 1592-1594, 2005.

3. Grill S, Rusterholz C, Zanetti-Dallenbach R, Tercanli S, Holzgreve W, Hahn S and Lapaire O: Potential markers of preeclampsia-a review. Reprod Biol Endocrinol 7: 70, 2009.

4. Huppertz B: Placental origins of preeclampsia: Challenging the current hypothesis. Hypertension 51: 970-975, 2008.

5. Oudejans CB and van Dijk M: Placental gene expression and pre-eclampsia. Placenta 29 (Suppl A): S78-S82, 2008.

6. Sitras V, Paulssen RH, Grønaas H, Leirvik J, Hanssen TA, Vårtun A and Acharya G: Differential placental gene expression in severe preeclampsia. Placenta 30: 424-433, 2009.

7. Founds SA, Conley YP, Lyons-Weiler JF, Jeyabalan A, Hogge WA and Conrad KP: Altered global gene expression in first trimester placentas of women destined to develop preeclampsia. Placenta 30: 15-24, 2009

8. Enquobahrie DA, Meller M, Rice K, Psaty BM, Siscovick DS and Williams MA: Differential placental gene expression in preeclampsia. Am J Obstet Gynecol 199: 566 e561-e511, 2008.

9. Lewis BP, Burge CB and Bartel DP: Conserved seed pairing, often flanked by adenosines, indicates that thousands of human genes are microRNA targets. Cell 120: 15-20, 2005.

10. Yu J, Wang F, Yang GH, Wang FL, Ma YN, Du ZW and Zhang JW: Human microRNA clusters: Genomic organization and expression profile in leukemia cell lines. Biochem Bioph Res Commun 349: 59-68, 2006.

11. Brennecke J, Hipfner DR, Stark A, Russell RB and Cohen SM: bantam encodes a developmentally regulated microRNA that controls cell proliferation and regulates the proapoptotic gene hid in Drosophila. Cell 113: 25-36, 2003.

12. Calame K: MicroRNA-155 function in B Cells. Immunity 27: 825-827, 2007.

13. Jopling CL, Yi M, Lancaster AM, Lemon SM and Sarnow P: Modulation of hepatitis C virus RNA abundance by a liver-specific MicroRNA. Science 309: 1577-1581, 2005.

14. Hu Y, Li P, Hao S, Liu L, Zhao J and Hou Y: Differential expression of microRNAs in the placentae of Chinese patients with severe pre-eclampsia. Clin Chem Lab Med 47: 923-929, 2009.

15. Mouillet JF, Chu T, Nelson DM, Mishima T and Sadovsky Y: MiR-205 silences MED1 in hypoxic primary human trophoblasts. FASEB J 24: 2030-2039, 2010.

16. Skrzypek K, Tertil M, Golda S, Ciesla M, Weglarczyk K, Collet G, Guichard A, Kozakowska M, Boczkowski J, Was $\mathrm{H}$, et al: Interplay between heme oxygenase-1 and miR-378 affects non-small cell lung carcinoma growth, vascularization and metastasis. Antioxid Redox Signal 19: 644-660, 2013.

17. Chen LT, Xu SD, Xu H, Zhang JF, Ning JF and Wang SF: MicroRNA-378 is associated with non-small cell lung cancer brain metastasis by promoting cell migration, invasion and tumor angiogenesis. Med Oncol 29: 1673-1680, 2012.

18. Corthorn J, Germain AA, Chacón C, Rey S, Soto GX, Figueroa CD, Müller-Esterl W, Duarte I and Valdés G: Expression of kallikrein, bradykinin b2 receptor and endothelial nitric oxide synthase in placenta in normal gestation, preeclampsia and placenta accreta. Endocrine 29: 491-499, 2006.

19. Li F, Hagaman JR, Kim HS, Maeda N, Jennette JC, Faber JE, Karumanchi SA, Smithies O and Takahashi N: eNOS deficiency acts through endothelin to aggravate sFlt-1-induced pre-eclampsia-like phenotype. J Am Soc Nephrol 23: 652-660, 2012. 
20. Mitchell PS, Parkin RK, Kroh EM, Fritz BR, Wyman SK, Pogosova-Agadjanyan EL, Peterson A, Noteboom J, O'Briant KC, Allen A, et al: Circulating microRNAs as stable blood-based markers for cancer detection. Proc Natl Acad Sci USA 105: 10513-10518, 2008.

21. Chim SS, Shing TK, Hung EC, Leung TY, Lau TK, Chiu RW and Lo YM: Detection and characterization of placental microRNAs in maternal plasma. Clin Chem 54: 482-490, 2008.

22. Gilad S, Meiri E, Yogev Y, Benjamin S, Lebanony D, Yerushalmi N, Benjamin H, Kushnir M, Cholakh H, Melamed N, et al: Serum microRNAs are promising novel biomarkers. PLoS One 3: e3148, 2008.

23. Wang K, Zhang S, Marzolf B, Troisch P, Brightman A, Hu Z Hood LE and Galas DJ: Circulating microRNAs, potential biomarkers for drug-induced liver injury. Proc Natl Acad Sci USA 106: 4402-4407, 2009.

24. Sibai B, Dekker G and Kupferminc M: Pre-eclampsia. Lancet 365: 785-799, 2005

25. Levine RJ, Lam C, Qian C, Yu KF, Maynard SE, Sachs BP, Sibai BM, Epstein FH, Romero R, Thadhani R, et al; CPEP Study Group: Soluble endoglin and other circulating antiangiogenic factors in preeclampsia. N Engl J Med 355: 992-1005, 2006.

26. Myatt L: Role of placenta in preeclampsia. Endocrine 19: 103-111, 2002.

27. Maynard S, Epstein FH and Karumanchi SA: Preeclampsia and angiogenic imbalance. Annu Rev Med 59: 61-78, 2008.

28. Pineles BL, Romero R, Montenegro D, Tarca AL, Han YM Kim YM, Draghici S, Espinoza J, Kusanovic JP, Mittal P, et al: Distinct subsets of microRNAs are expressed differentially in the human placentas of patients with preeclampsia. Am J Obstet Gynecol 196: 261 e261-e266, 2007.

29. Roman H, Marpeau L and Hulsey TC: Surgeons' experience and interaction effect in randomized controlled trials regarding new surgical procedures. Am J Obstet Gynecol 199: 108 e101-e106, 2008.

30. Hu Y, Li P, Hao S, Liu L, Zhao J and Hou Y: Differential expression of microRNAs in the placentae of Chinese patients with severe pre-eclampsia. Clin Chem Lab Med 47: 923-929, 2009.
31. Guo G, Lade JA, Wilton AN, Moses EK, Grehan M, Fu Y, Qiu H, Cooper DW and Brennecke SP: Genetic susceptibility to pre-eclampsia and chromosome 7q36. Hum Genet 105: 641-647, 1999.

32. Demircubuk AG, Coskun MY, Demiryurek S, Dokuyucu R, Öztuzcu S, Taviloğlu ZȘ, Arslan A and Sivaslı E: Endothelial NOS gene Glu298Asp polymorphism in preterm neonates with respiratory distress syndrome. Pediatr Pulmonol 48: 976-980, 2013.

33. Duplain H, Burcelin R, Sartori C, Cook S, Egli M, Lepori M, Vollenweider P, Pedrazzini T, Nicod P, Thorens B, et al: Insulin resistance, hyperlipidemia and hypertension in mice lacking endothelial nitric oxide synthase. Circulation 104: 342-345, 2001.

34. Xiang W, Chen H, Hu L and Xu X: Endothelial nitric oxide synthase traffic inducer in the umbilical vessels of the patients with pre-eclampsia. J Huazhong U Sci Technolog Med Sci 29: 243-245, 2009

35. SoundararajanR and Rao AJ: Trophoblast 'pseudo-tumorigenesis': Significance and contributory factors. Reprod Biol Endocrinol 2: $15,2004$.

36. Lala PK and Chakraborty C: Factors regulating trophoblast migration and invasiveness: Possible derangements contributing to pre-eclampsia and fetal injury. Placenta 24: 575-587, 2003.

37. Buhimschi I, Yallampalli C, Chwalisz K and Garfield RE: Pre-eclampsia-like conditions produced by nitric oxide inhibition: Effects of L-arginine, D-arginine and steroid hormones. Hum Reprod 10: 2723-2730, 1995.

38. Enquobahrie DA, Abetew DF, Sorensen TK, Willoughby D, Chidambaram K and Williams MA: Placental microRNA expression in pregnancies complicated by preeclampsia. Am J Obstet Gynecol 204: 178 e112-e121, 2011.

39. Wang H, Li M, Zhang R, Wang Y, Zang W, Ma Y, Zhao G and Zhang G: Effect of miR-335 upregulation on the apoptosis and invasion of lung cancer cell A549 and H1299. Tumour Biol 34: 3101-3109, 2013.

40. Ueno K, Hirata H, Shahryari V, Chen Y, Zaman MS, Singh K, Tabatabai ZL, Hinoda Y and Dahiya R: Tumour suppressor microRNA-584 directly targets oncogene Rock-1 and decreases invasion ability in human clear cell renal cell carcinoma. $\mathrm{Br}$ J Cancer 104: 308-315, 2011.

41. Caterina MJ and Devreotes PN: Molecular insights into eukaryotic chemotaxis. FASEB J 5: 3078-3085, 1991. 